Research Article

\title{
Comparative genome analysis of the SPL gene family reveals novel evolutionary features in maize
}

\author{
Xiaojian Peng ${ }^{1 *}$, Qianqian Wang ${ }^{2 *}$, Yang Zhao ${ }^{1}$, Xiaoyu $\mathrm{Li}^{1}$ and Qing $\mathrm{Ma}^{1}$ \\ ${ }^{1}$ National Engineering Laboratory of Crop Stress Resistance, Key Laboratory of Crop Biology of Anhui \\ Province, School of Life Sciences, Anhui Agricultural University, Hefei, China. \\ ${ }^{2}$ Institute of Horticulture, Anhui Academy of Agricultural Sciences, Hefei, China.
}

\begin{abstract}
SPLS are plant-specific transcription factors that play important regulatory roles in plant growth and development. Systematic analysis of the SPL family has been performed in numerous plants, such as Arabidopsis, rice, and Populus. However, no comparative analysis has been performed across different species to examine evolutionary features. In this study, we present a comparative analysis of SPLS in different species. The results showed that 84 $S P L S$ of the four species can be divided into six groups according to phylogeny. We found that most of the SPL-containing regions in maize showed extensive conservation with duplicated regions of rice and sorghum. A gene duplication analysis in maize indicated that $Z m S P L s$ showed a significant excess of segmental duplication. The $\mathrm{Ka} / \mathrm{Ks}$ analysis indicated that 9 out of 18 duplicated pairs in maize experienced positive selection, while SPL gene pairs of rice and sorghum mainly evolved under purifying selection, suggesting novel evolutionary features for ZmSPLs. The $31 \mathrm{ZmSPLs}$ were further analyzed by describing their gene structure, phylogenetic relationships, chromosomal location, and expression, Among the ZmSPLs, 13 were predicated to be targeted by miR156s and involved in drought stress response. These results provide the foundation for future functional analyses of ZmSPLs.
\end{abstract}

Keywords: SPL, phylogenetic relationship, gene duplication, miR156 expression.

Received: June 8, 2017; Accepted: October 4, 2018.

\section{Introduction}

Transcription factors (TFs) are a large class of regulators controling gene expression by activating or repressing target genes at the transcriptional level. Increasing evidence indicates that TFs have important roles in the regulating networks of plant growth and development processes (Riechmann et al., 2001). SPLs (SQUAMOSA promoter binding protein-like) comprise a family of plant-specific transcription factors that contain a highly conserved SBP domain consisting of about 76 amino acids (Chen et al., 2010). This domain has been implicated in DNA binding and nuclear localization, and also features two zinc-binding sites assembled as Cys-Cys-Cys-His and Cys-Cys-HisCys, respectively (Klein et al., 1996; Yamasaki et al., 2004). Gene structure analysis indicated that the nuclear localization signal (NLS) region partially overlapped with the second Zn-finger located at the C-terminal of the SBP domain. SBP-domain encoding proteins were firstly iso-

Send correspondence to Qing Ma. National Engineering Laboratory of Crop Stress Resistance, Key Laboratory of Crop Biology of Anhui Province, School of Life Sciences, Anhui Agricultural University, No. 130, Changjiang West Road, Hefei 230036, China. E-mail: maqingahau2016@163.com.

'These authors contributed equally to this work. lated from Antirrhinum majus designated as AmSBP1 and AmSBP2. These two proteins can recognize a conserved motif in the promoter region of the floral meristem identity gene SQUAMOSA, which is a member of the MADS-box gene family based on its in vitro binding activity (Klein et al., 1996). Subsequent experiments indicated that the palindromic GTAC core motif of the cis-element is essential for efficient DNA binding by different SBP proteins (Birkenbihl et al., 2005; Cardon et al., 1997). To date, the SPL gene family has been identified in various plant genomes, such as Arabidopsis, rice, and Populus (Cardon et al., 1999; Xie et al., 2006; Guo et al., 2008; Li and Lu 2014).

In Arabidopsis, a total of 16 members have been identified as SPL proteins. Several biological experiments demonstrated that SPL proteins have important functions in plant development processes, especially flower development. For example, the AtSPL3 gene was shown to be involved in the floral transition, and it was the first SPL gene identified in Arabidopsis. As an ortholog of SQUAMOSA, $A t S P L 3$ can interact with the promoter region of the floral meristem identity gene APETALA1 (AP1), and constitutive expression of this gene in Arabidopsis can result in an early flowering phenotype (Cardon et al., 1997). Loss-offunction mutation of the Arabidopsis SPL8 gene indicated 
that AtSPL8 can regulate pollen sac development (Unte et al., 2003). In maize, the tasselsheath4 (tsh4) mutant of an SPL gene was shown to regulate bract development and the establishment of meristem boundaries (Chuck et al., 2010). In addition, SPL genes (SPLS) were also demonstrated to play crucial roles in fruit development (Manning et al., 2006), leaf development (Stone et al., 2005), plant hormone signaling (Zhang et al., 2007), male fertility (Xing, 2010), and shoot development (Wu and Poethig, 2006).

Besides transcription factors, miRNAs are another other class of important regulators of gene expression, acting at the post-transcriptional level (Lee et al., 1993; Zhang et al., 2006). These small RNA molecules (20-24 nucleotides in length) can cause the degradation of mRNAs or repress translation by binding to the mRNAs of the target genes (Zhang et al., 2006). Most of the miRNAs in plants are evolutionarily conserved, encoded by gene families (Jones-Rhoades et al., 2006). Among them, miR156/157, a miRNA family that is highly conserved in plants (Axtell and Bowman, 2008), is thought to be involved in important developmental processes. Previous studies demonstrated that half of the SPLs have been found to be targeted by miR156/157 family. For example, 10 of the 16 Arabidopsis SPLs, were targeted by the miR156 family (Rhoades et al., 2002; Schwab et al., 2005; Wu and Poethig, 2006; Wang et al., 2009; Yu et al., 2010). In rice, 11 of the 19 SPLs were found to be regulated by OsmiR156 (Xie et al., 2006).

Despite the progress in function studies of SPLs in many species, no comparative analysis has been reported across different species to study the evolution and functional relevance of this family. Although the maize SPL gene family has been reported by Hultquist and Dorweiler (Dorweiler, 2008), our understanding of this gene family in maize is still rather limited. Therefore, we firstly performed a comparative analysis of this family to dissect the evolutionary features in different species, and $31 \mathrm{ZmSPLs}$ were further characterized, including gene structure, phylogenetic relationships, gene duplication, amongst others. Quantitative real-time PCR (RT-qPCR) analysis was performed to examine the expression pattern of miR156 targeted genes in different tissues and in response to drought stress. These results contribute to a basic understanding of the SPL gene family in different species, and provide a foundation to further elucidate the SPL gene function in maize.

\section{Material and Methods}

\section{Whole-genome identification and phylogenetic analysis of SPLS}

To identify maize SPL proteins, the Hidden Markov Model (HMM) profile of the SBP domain (PF03110.7) retrieved from Pfam database (http://pfam.xfam.org/) (Finn et al., 2006) was adopted as query against maize genome database (http://www.maizesequence.org/index.html), with an cutoff E-value of $\mathrm{le}^{-3}$. Sequences of Arabidopsis and rice SPL proteins were also used to query against the maize genome to identify all possible maize SPL proteins (Cardon et al., 1999; Xie et al., 2006; Guo et al., 2008). The candidate sequences that met the standards were confirmed again by Pfam database and SMART (http://smart.emblheidelberg.de/) (Letunic, 2009). Finally, redundant sequences were removed manually after alignments using MUSCLE software (Edgar, 2004). To identify sorghum $S P L s$, the complete genome sequence of sorghum was obtained (ftp://ftp.ensemblgenomes.org/pub/plants/release31/fasta/sorghum_bicolor/pep/), and the same method as described above was adopted. To understand the evolutionary relationships of the SPL family, full-length sequences of the SPL proteins were aligned using MUSCLE software. A phylogenetic tree was constructed using MEGA v4.0 (Tamura, 2007) by the neighbor-joining (NJ) method with 1,000 bootstrap replicates.

\section{Synteny analysis, gene duplication, and evolution analysis}

Syntenic blocks among maize, rice, and sorghum were evaluated by MCScan software (Wang et al., 2012) and alignments with an E-value of $1 \mathrm{e}^{-5}$ were considered significant matches. Then, the duplicated SPLs from these syntenic blocks were identified using a Perl script, and the relationships of the duplicated genes, including segmental and tandem duplications, were finally visualized using Circos (http://circos.ca) (Krzywinski et al., 2009; Wang et al., 2015). DnaSP v5.0 (Rozas et al., 2003) was used to estimate the number of nonsynonymous substitutions per nonsynonymous site $(\mathrm{Ka})$ and synonymous substitution per synonymous site $(\mathrm{Ks})$ of the duplicated genes. The $\mathrm{Ka} / \mathrm{Ks}$ ratios between duplicated genes were analyzed to deduce the selection model. To obtain further insight into selection pressure among duplicated gene pairs, a sliding window analysis of the $\mathrm{Ka} / \mathrm{Ks}$ ratios was conducted with the following parameters: window size $150 \mathrm{bp}$ and step size $9 \mathrm{bp}$. For duplication time analysis, the Ks value was translated into duplication time in million years based on a synonymous mutation rate of $\lambda$ substitutions per synonymous site per year, as $\mathrm{T}=\mathrm{Ks} / 2 \lambda 10^{-6}$ million years ago (Mya) $\left(\lambda=6.510^{-9}\right.$ for grasses) (Gaut et al., 1996; Quraishi et al., 2011).

\section{Sequence analysis and chromosomal locations of ZmSPL genes}

Information regarding the exon number, open reading frame (ORF) length, molecular weight $(\mathrm{kDa})$, and isoelectric point (pI) of maize SPL proteins were determined by the Expasy program (http://www.expasy.org/tools/). Gene structure was predicted through alignments of the coding sequences (CDS) with corresponding genomic sequences using GSDS (http://gsds.cbi.pku.edu.cn/) (Hu et al., 2015). Conserved motifs were investigated by MEME (Multiple Expectation Maximization for Motif Elicitation) (Bailey and Elkan, 1995) with the parameters used in our 
previous study (Zhao et al., 2011). The chromosome location image was generated by MapInspect software (http://www.plantbreeding.wur.nl/uk/soft-

ware_mapinspect.html) according to the starting positions of ZmSPLs on the 10 chromosomes.

\section{Prediction of $Z m S P L$ genes targeted by miR156}

To predict ZmSPLs regulated by miR156, the sequence of maize miR156 was first obtained from miRBase (http://www.mirbase.org/) (Kozomara and Griffithsjones, 2010). Then, ZmSPLs targeted by miR156 were predicted by searching the coding regions and 3' UTRs of all SPLs for complementary sequences to the maize miR156 sequence using psRNATarget server with default parameters (http://plantgrn.noble.org/psRNATarget/?function=3) (Dai and Zhao, 2011).

\section{Expression pattern analysis using transcriptome data}

Transcriptome data of the genome-wide gene expression atlas of the maize inbred line B73 was used to elucidate the expression pattern of ZmSPLs during different development stages (Sekhon et al., 2013). A heat map was generated based on the FPKM (fragments per kilobase of exon per million fragments mapped) values, which were initially transformed by taking $\log 2(\mathrm{FPKM}+1)$ and then loaded into $\mathrm{R}$ and the Bioconductor program (http://www.bioconductor.org/) (Ross and Robert, 2008).

\section{Plant materials, stress treatments, RNA extraction, and RT-qPCR analysis}

To examine the expression profile during different developmental stages, four representative tissues, including root, leaf, stem, and silk were collected from a life cycle of the maize inbred line B73. For stress treatment, maize seeds were surface-sterilized in 1 (v/v) Topsin-M (Rotam Crop Sciences Ltd.) for $10 \mathrm{~min}$, washed in deionized water, and germinated on wet filter paper at $28{ }^{\circ} \mathrm{C}$ for 3 days. The germinated seeds were transplanted to enriched soil (turf to vermiculite in a ratio of 1:1) and grown in a greenhouse with a 14 -h light/10-h dark cycle at $28-30{ }^{\circ} \mathrm{C}$. Drought stress was performed by withholding watering at the threeleaf stage of maize seedlings. The seedling leaves were collected at $0,1,2$, and 4 days after treatment with relative leaf water content (RLWC) decreased to $98,70,60$, and 58\%, which represented normal plants, slight, moderate, and severe stresses, respectively. For all the stages, three biological replicates were performed for each sample. For RNA isolation, all the collected samples were extracted using Trizol reagent (Invitrogen). To remove possible contaminating genomic DNA, the extracted RNAs were treated with DNase I (Invitrogen) for $20 \mathrm{~min}$, then cDNAs were synthesized from $1 \mu \mathrm{g}$ of total RNA using the PrimerScript RT Master mix (TaKaRa). For RT-qPCR analysis, genespecific primers for maize SPL genes were designed using
Primer Express 3.0 software (Applied Biosystems), listed in Table S1, and the PCR assays and data analysis were performed as described previously (Peng et al., 2012).

Primer specificity were examined through the Primer Blast at NCBI, and their efficiency was tested by ordinary PCR. Amplification products were analyzed by agarose gel electrophoresis, and each primer pair was seen to amplified only one $100 \mathrm{bp}$ product, which indicated that these primers were suitable for RT-qPCR. The RT-qPCRs were performed in an ABI 7300 Real-Time machine, with a total reaction volume of $20 \mu \mathrm{L}$, containing SYBR Green Master Mix reagent, cDNA sample, primers and RNase-free water. The PCR run program was as follows: denaturation $\left(95^{\circ} \mathrm{C}\right.$ for $10 \mathrm{~min}$ ), amplification and quantification (40 cycles of $95^{\circ} \mathrm{C}$ for $15 \mathrm{~s}$ and $60^{\circ} \mathrm{C}$ for $1 \mathrm{~min}$ ), melting curve analysis $\left(60-95{ }^{\circ} \mathrm{C}\right.$, with a heating rate of $\left.0.3{ }^{\circ} \mathrm{C} / \mathrm{s}\right)$. The ZmActin gene was used for data normalization, and for each sample three technical replicates were performed. Relative expression levels were calculated using the comparative delta delta cycle threshold $(\Delta \Delta \mathrm{ct})$ method. The SPSS 19.0 software (http://www.spss.com.cn/) was used for statistical analysis.

\section{Results}

\section{SPL genes in different species}

In previous studies, a total of 19, 16, and 31 SPLS were identified in rice, Arabidopsis, and maize, repectively (Cardon et al., 1999; Xie et al., 2006; Dorweiler, 2008; Guo et al., 2008). Due to maize genome database updates, we performed a BlastP search against the genome database to identify maize SPLs using the Hidden Markov Model (HMM) profile of the SPL domain, and the same strategy was used to identify sorghum SPLs. By this approach, a total of 31 and 18 non-redundant sequences in maize and sorghum were identified after searching against Pfam and SMART, respectively. The total number of SPLs in maize was the same as in a previous study. In addition, the number of sorghum SPLS was similar to that in rice and Arabidopsis. These genes were named ZmSPL1-ZmSPL31 and SbSPL1-SbSPL18 according to their order of distribution on the chromosomes (Tables S2, S3). It should be noted that the number of SPLS in the maize genome was greater than that in rice, Arabidopsis, and sorghum. This gives rise to the question, as to where did these additional genes originally come from in the maize genome. To elucidate the possible mechanism(s) of this phenomenon, we subsequently performed a comparative analysis of SPL gene family in these species.

\section{Phylogenetic relationships of SPLs}

To examine the evolutionary relationships of SPLS among different plant species, full-length sequences of the SPL proteins were aligned using MUSCLE, and then a combined phylogenetic tree of 84 SPL protein sequences 
from the four species, including 31 of maize, 19 of rice, 18 of sorghum, and 16 of Arabidopsis, was constructed using the NJ method with 1000 bootstrap replicates (Figure 1).
The 84 SPLS were divided into six subfamilies (I-VI) according to phylogenetic relationship (bootstrap value $>$ $50 \%)$. Although each of the subfamilies contained repre-

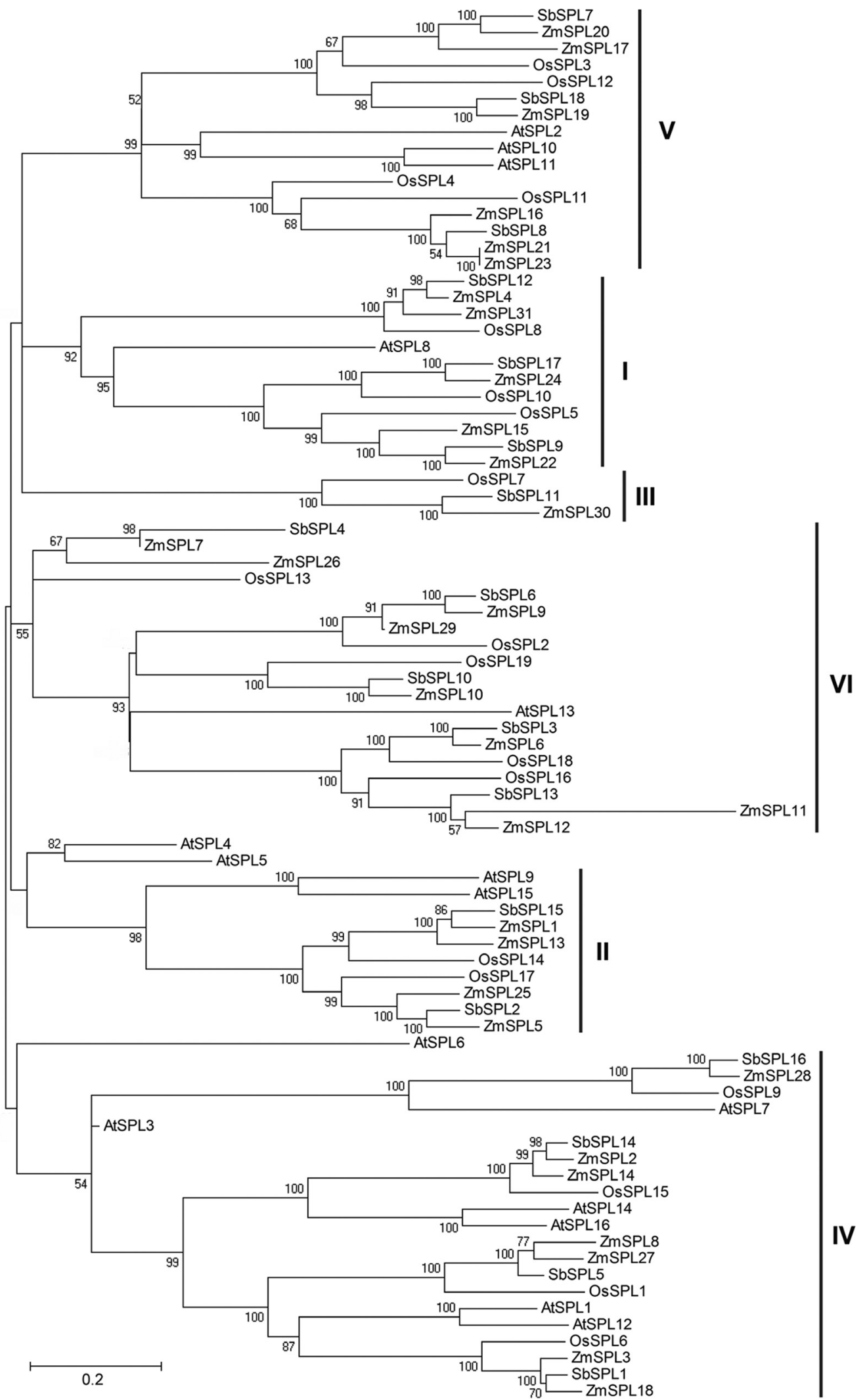

Figure 1 - Phylogenetic relationships of maize, rice, sorghum, and Arabidopsis SPL proteins. The phylogenetic tree was constructed using MEGA4.0 with the NJ method. Bootstrap values above $50 \%$ are shown at each node. 
sentative of rice, sorghum, and Arabidopsis SPLs, most maize SPLs showed closer relationships with sorghum SPLs than rice and Arabidopsis, suggesting a closer evolutionary relationship of the two species. For example, a total of 16 orthologous pairs were identified between maize and sorghum. We noted that the number of SPLS located in different subfamilies had a significant difference, ranging from 3 (III) to 20 (IV). Most of the members located in the same phylogenetic clade had well-supported bootstrap values, while some proteins showed unclear evolutionary relationships with lower bootstrap values, such as AtSPL4, AtSPL5, and AtSPL6. We also noted that the numbers of maize SPL proteins in most of the six groups were higher than other species, suggesting SPLs had especially expanded in the maize genome.

\section{Synteny analysis of SPLs among maize, sorghum, and rice}

To examine the origin and evolutionary history of SPLs among maize, sorghum, and rice, a comparative analysis was performed to identify SPL orthologous pairs. Because Arabidopsis belongs to the Dicotyledoneae group of plants, orthologous pairs were not detected with the three other species. Through the comparative analysis of the genomic regions hosting the SPLs using MCScan software, we observed strongly conserved synteny among the three species. A total of 104 orthologous gene pairs were found among maize, rice, and sorghum, including 38 pairs between maize and rice, 36 pairs between maize and sorghum, and 30 pairs between sorghum and rice (Figure 2, Table S4). The numbers of orthologous gene pairs among

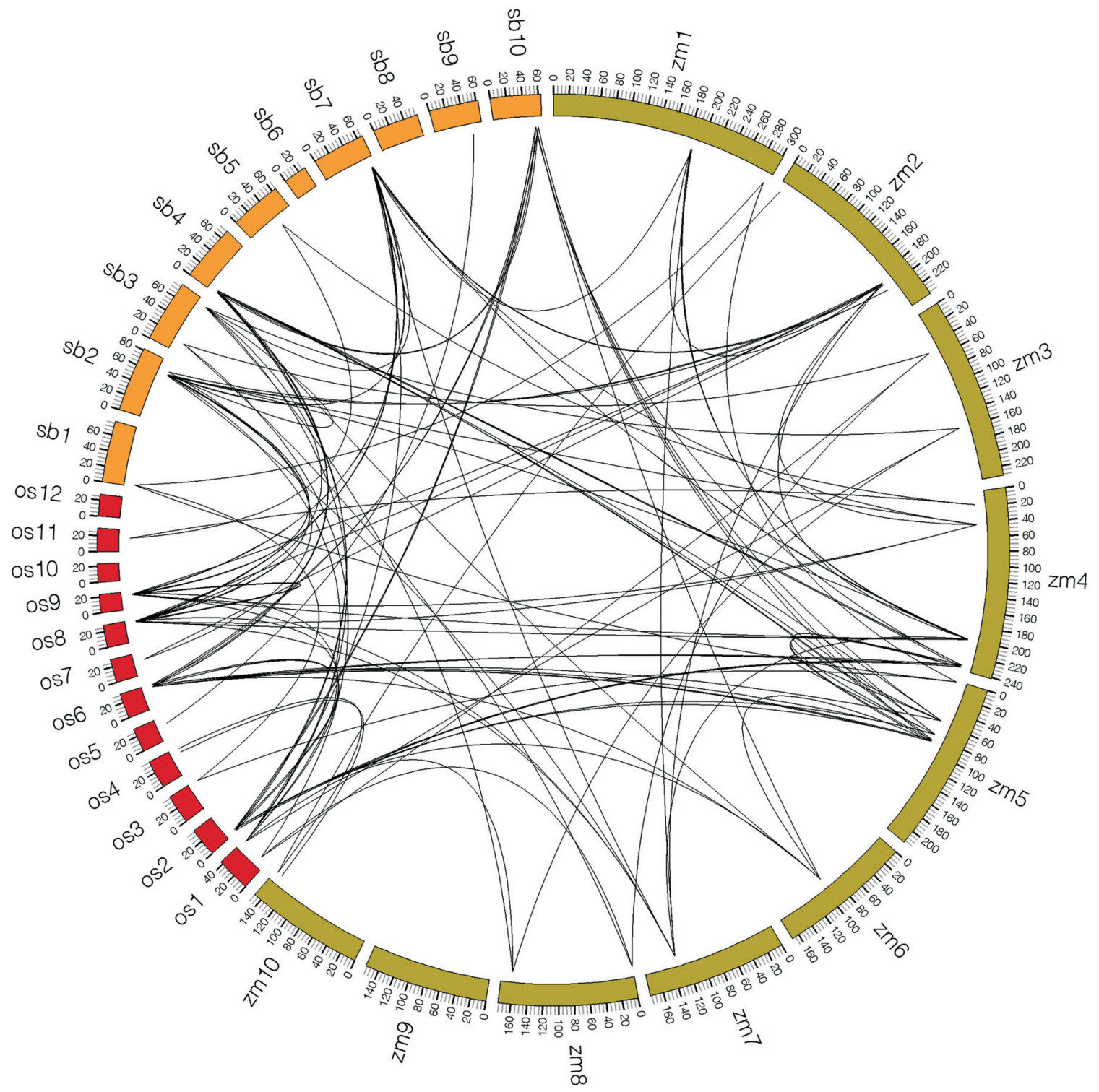

Figure 2 - Synteny analysis of 68 SPLs from maize, sorghum, and rice. Maize, sorghum and rice chromosomes were labeled zm, sb, and os by different color boxes, respectively. The numbers along each chromosome box indicate sequence length of each chromosome in megabases. Black lines represent the syntenic relationships of orthologous gene pairs. 
the three plants were similar, suggesting the conserved evolution of the SPL family. Some differences were also observed among the three species, for example, the ZmSPL16 and ZmSPL17 had two orthologous genes in rice (ZmSPL16/OsSPL4, OSSPL11; ZmSPL17/OSSPL3, OSSPL12), while only one was identified in sorghum (ZmSPL16/SbSPL8; ZmSPL17/SbSPL7), respectively, which might be related to gene loss in the evolution of sorghum. In addition, the syntenic information also provided important clues to study the putative function of the collinear gene. For example, ZmSPL4 encoding the $l g 1$ gene (Moreno et al., 1997) had one collinear gene in rice $(O S S P L 8)$ as well as in sorghum (SbSPL12). Especially, ZmSPL11 encoding the tgal gene (Wang et al., 2005) had two orthologous genes in rice (OSSPL16 and OSSPL18) and sorghum (SbSPL3 and SbSPL13). These genes existing in different species might have originated from a common ancestor, which might share a similar regulatory role in plant growth and development.

\section{Gene duplication of SPLS}

The number of ZmSPLs (31) was almost twice that of Arabidopsis (16), and also much higher than that in rice (19) and sorghum (18) (Cardon et al., 1999; Xie et al., 2006). Gene duplication, including tandem and segmental duplications, are thought to have played important roles in the amplification of gene families in animals and plants (Moore and Purugganan, 2003). Thus, potential duplication events were analyzed to reveal the mechanism(s) behind the expansion of the maize SPL family. According to the syntenic regions and phylogenetic analysis, $18 \mathrm{ZmSPL}$ gene pairs ( 24 genes) were located on the segmental duplication regions, accounting for $77.4 \%$ of the number of ZmSPLs (Figure 3, Table 1). In sorghum, six gene pairs (nine genes) were localized on the segmental duplication regions, accounting for $50 \%$ of the sorghum SPLS. In rice, 11 members forming seven gene pairs were detected, which accounted for $57.8 \%$ of the rice SPLs. In addition, no significant tandem duplication events were detected among the three plants. These findings indicated that segmental duplication was the major factor that contributed to the expansion of SPL gene family, especially for maize.

To further understand the duplication and divergence of SPLs, the Ka, Ks, and $\mathrm{Ka} / \mathrm{Ks}$ ratio were calculated for each duplicated pair. The Ka and Ks results were used to examine the course of divergence after duplication, and the $\mathrm{Ka} / \mathrm{Ks}$ ratio was applied to explore different selective constrains. Generally, a $\mathrm{Ka} / \mathrm{Ks}$ ratio $<1$ means purifying selection, a ratio $=1$ indicates neutral selection, while a ratio $>1$ stands for positive selection (Lynch and Conery, 2000). The results showed that the $\mathrm{Ka} / \mathrm{Ks}$ ratio of the 18 duplicated ZmSPLs pairs ranged from 0.449 to 1.605 . Among them, nine duplicated pairs had a $\mathrm{Ka} / \mathrm{Ks}$ ratio $<1$. Moreover, the values of ZmSPL13/-5, ZmSPL15/-22 and ZmSPL22/-24 were less than 0.6 , which suggests strong purifying selection during evolution. The other nine pairs showed a Ka/Ks ratio $>1$, indicating that these gene pairs evolved under positive selection (Table 1). In rice and sorghum, the $\mathrm{Ka} / \mathrm{Ks}$ ratios of all gene pairs were $<1$, except for OSSPL2/-18, suggesting that these gene pairs mainly evolved under purifying selection. To obtain further insight into the selection pressure of different sites/regions, we performed a sliding-window analysis of the $\mathrm{Ka} / \mathrm{Ks}$ ratio for each duplicated gene pair. As shown in Figure 4, numerous sites/regions showed evidence of strong positive selection, especially for $Z m S P L$ gene pairs. In contrast, the other sites/regions were conserved under purifying selection, such as OSSPL14/-17 and $\operatorname{SbSPL2/-15.}$

According to the estimation for Ks, the dates for 31 segmental duplication pairs of maize, rice, and sorghum, were calculated based on a rate of $6.510^{-9}$ substitutions per site per year (Gaut et al., 1996; Quraishi et al., 2011). The results indicated that the 18 maize duplication events were estimated to have occurred approximately between 4.81 to
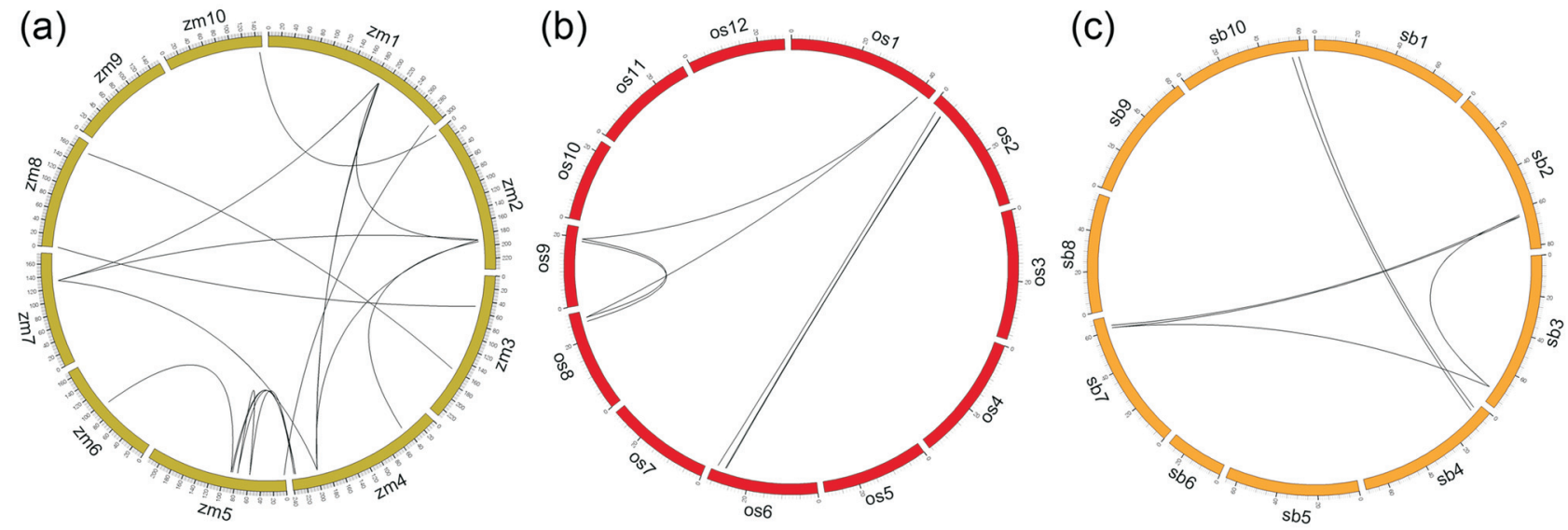

Figure 3 - Synteny analysis of maize (a), rice 9 (b), and sorghum (c) SPLs. Maize, sorghum, and rice chromosomes were labeled zm, sb and os by different color boxes, respectively. The number along each chromosome box indicate sequence length of each chromosome in megabases. Black lines represent the syntenic relationships between SPLs. 
Table $1-\mathrm{Ka} / \mathrm{Ks}$ analysis and estimated divergence time for the duplicated SPL paralogs

\begin{tabular}{|c|c|c|c|c|c|c|}
\hline Duplicated pairs & $\mathrm{Ka}$ & Ks & $\mathrm{Ka} / \mathrm{Ks}$ & Purifying selection & Date (Mya) & Duplicate type \\
\hline ZmSPL1-ZmSPL13 & 0.135 & 0.164 & 0.822 & Yes & 12.61 & Segmental \\
\hline ZmSPL5-ZmSPL25 & 0.122 & 0.126 & 0.966 & Yes & 9.68 & Segmental \\
\hline ZmSPL1-ZmSPL5 & 0.394 & 0.395 & 0.997 & Yes & 30.39 & Segmental \\
\hline ZmSPL1-ZmSPL25 & 0.374 & 0.344 & 1.088 & No & 26.45 & Segmental \\
\hline ZmSPL13-ZmSPL5 & 0.346 & 0.651 & 0.532 & Yes & 50.08 & Segmental \\
\hline ZmSPL13-ZmSPL25 & 0.373 & 0.279 & 1.337 & No & 21.45 & Segmental \\
\hline ZmSPL2-ZmSPL14 & 0.100 & 0.063 & 1.605 & No & 4.81 & Segmental \\
\hline ZmSPL3-ZmSPL18 & 0.083 & 0.073 & 1.136 & No & 5.64 & Segmental \\
\hline ZmSPL4-ZmSPL31 & 0.145 & 0.093 & 1.559 & No & 7.14 & Segmental \\
\hline ZmSPL6-ZmSPL11 & 0.480 & 0.565 & 0.849 & Yes & 43.49 & Segmental \\
\hline ZmSPL8-ZmSPL27 & 0.114 & 0.122 & 0.934 & Yes & 9.35 & Segmental \\
\hline ZmSPL9-ZmSPL29 & 0.124 & 0.100 & 1.242 & No & 7.65 & Segmental \\
\hline ZmSPL15-ZmSPL22 & 0.173 & 0.385 & 0.449 & Yes & 29.63 & Segmental \\
\hline ZmSPL22-ZmSPL24 & 0.365 & 0.611 & 0.598 & Yes & 46.98 & Segmental \\
\hline ZmSPL16-ZmSPL21 & 0.101 & 0.085 & 1.178 & No & 6.56 & Segmental \\
\hline ZmSPL17-ZmSPL20 & 0.257 & 0.272 & 0.948 & Yes & 20.88 & Segmental \\
\hline ZmSPL17-ZmSPL19 & 0.553 & 0.526 & 1.051 & No & 40.46 & Segmental \\
\hline ZmSPL20-ZmSPL19 & 0.440 & 0.394 & 1.118 & No & 30.30 & Segmental \\
\hline SbSPL2-SbSPL15 & 0.228 & 0.467 & 0.488 & Yes & 35.923 & Segmental \\
\hline SbSPL3-SbSPL6 & 0.406 & 0.534 & 0.760 & Yes & 41.08 & Segmental \\
\hline SbSPL3-SbSPL13 & 0.2060 .2061 & 0.474 & 0.435 & Yes & 34.46 & Segmental \\
\hline SbSPL6-SbSPL13 & 0.426 & 0.597 & 0.714 & Yes & 45.92 & Segmental \\
\hline SbSPL18-SbSPL7 & 0.265 & 0.611 & 0.433 & Yes & 47.00 & Segmental \\
\hline SbSPL17-SbSPL9 & 0.306 & 0.419 & 0.730 & Yes & 32.23 & Segmental \\
\hline OSSPL2-OSSPL16 & 0.380 & 0.519 & 0.732 & Yes & 39.92 & Segmental \\
\hline OSSPL2-OSSPL18 & 0.4960 .496 & 0.496 & 1.000 & No & 38.15 & Segmental \\
\hline OSSPL3-OSSPL12 & 0.487 & 0.524 & 0.929 & Yes & 40.31 & Segmental \\
\hline OSSPL4-OSSPL11 & 0.317 & 0.535 & 0.593 & Yes & 41.15 & Segmental \\
\hline OSSPL5-OSSPL10 & 0.338 & 0.412 & 0.820 & Yes & 31.69 & Segmental \\
\hline OsSPL14-OsSPL17 & 0.185 & 0.450 & 0.411 & Yes & 34.62 & Segmental \\
\hline OsSPL16-OSSPL18 & 0.292 & 0.461 & 0.633 & Yes & 35.46 & Segmental \\
\hline
\end{tabular}

50.08 Mya (Table 1), and the duplication events of rice and sorghum SPLs were estimated to have occurred between 34.46 to 47.00 Mya.

\section{Sequence analysis of maize SPLS}

Molecular weight (MW) and isoelectric point (pI) of the $31 \mathrm{ZmSPLs}$ were determined using the Expasy server. The results showed that the ZmSPL proteins had a large variation in the length (bp) of the open reading frame (ranging from 300 to 3,339 bp) (Table S2). The $31 \mathrm{ZmSPLs}$ were divided into six subfamilies based on the unrooted NJ tree (Figure S1a). Gene structure analysis indicated that the maize SPL family had highly diverse distributions of exon regions (Figure S1b). However, most SPLs within the same subfamilies of the phylogenetic tree had a similar gene structure. A total of 20 conserved motifs were identified in the maize SPL proteins (Table S5). Compared with the phylogenetic analysis, we found that genes located in the same subfamily had similar motif compositions (Figure $\mathrm{S} 2$ ). According to the starting positions of the maize SPL genes annotated by the maize B73 genome database, chromosome location analysis indicated that all of the 31 ZmSPLs were mapped to 9 of the 10 chromosomes with a clear non-random distribution (Figure S3) with approximately $45 \%$ of the SPLS on chromosome 4 (eight genes) and 5 (six genes).

\section{Identification of ZmSPLs targeted by miR156}

A series of SPLs have been confirmed to be targeted by miR156 in Arabidopsis, grape, and Populus. In general, the complementary sites of miR156 tend to be completely conserved and to locate in the coding regions or 3' UTRs of 

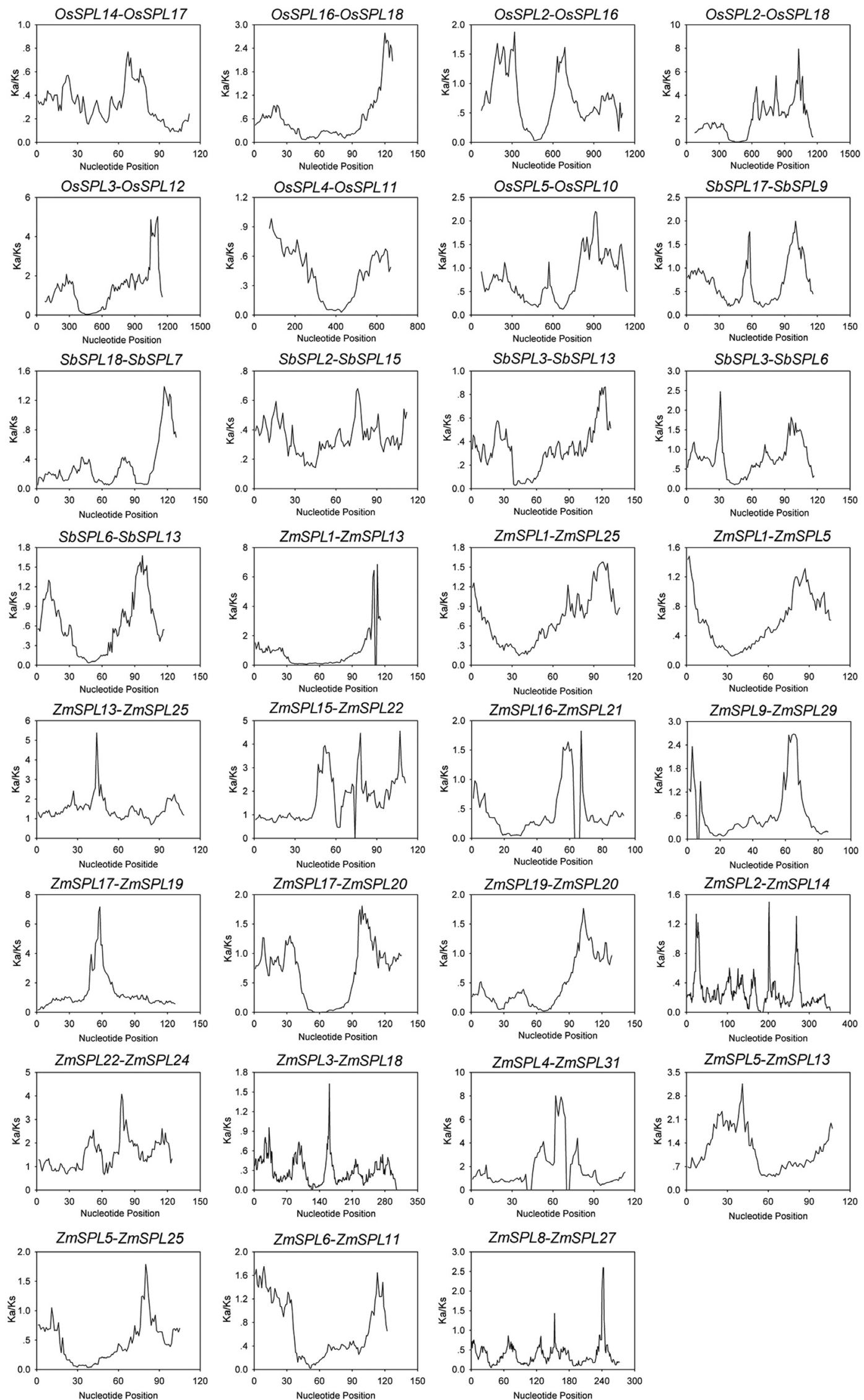

Figure 4 - Sliding window plots of segmental duplicated SPLs. Window size is $150 \mathrm{bp}$, and step size is $9 \mathrm{bp}$. 
SPLs in different plants (Schwarz et al., 2008; Hou et al., 2013; Li and Lu, 2014;). To identify the ZmSPLs targeted by miR156, we searched the coding regions and 3' UTRs of all ZmSPLs for targets of maize miR156 using the psRNATarget online prediction tool with default parameters (Dai and Zhao, 2011). A total of 13 ZmSPLs were predicted to be potential targets of miR156 (Figure 5). We also found that the targeting sites of miR156 were located in coding regions for $11 \mathrm{ZmSPLs}$, and only two complementary sites were located in the 3' UTRs (ZmSPL7 and ZmSPL26). Consistent with previous studies, the targeting sites of maize SPLs were highly conserved in the evolution by the alignments of miR 156 with their complementary sequence of maize SPLs (Figure 6).

\section{Expression patterns of $Z m S P L$ genes in different developmental stages}

The transcriptome data of the genome-wide gene expression atlas of maize was used to analyze the expression patterns of SPLs in different developmental stages (Sekhon et al., 2013) (Figure 7). The results showed that most ZmSPLs had ubiquitously expression in the 18 different tissues. The group IV members seem to play regulatory roles in maize at multiple development stages based on the constitutive expression at relatively high level in all of the 18 tissues. On the contrary, the group I genes were only expressed in one or a few tissues and at a very low expression level, for example, ZmSPL22 and ZmSPL31 are merely expressed in V3_Stem and SAM. Furthermore, ZmSPL15 was not expressed among the 18 tissues. By comparing the expression patterns of the duplicated gene pairs, we found that most of the duplicated gene pairs had similar expression patterns, but some with obvious divergence were also

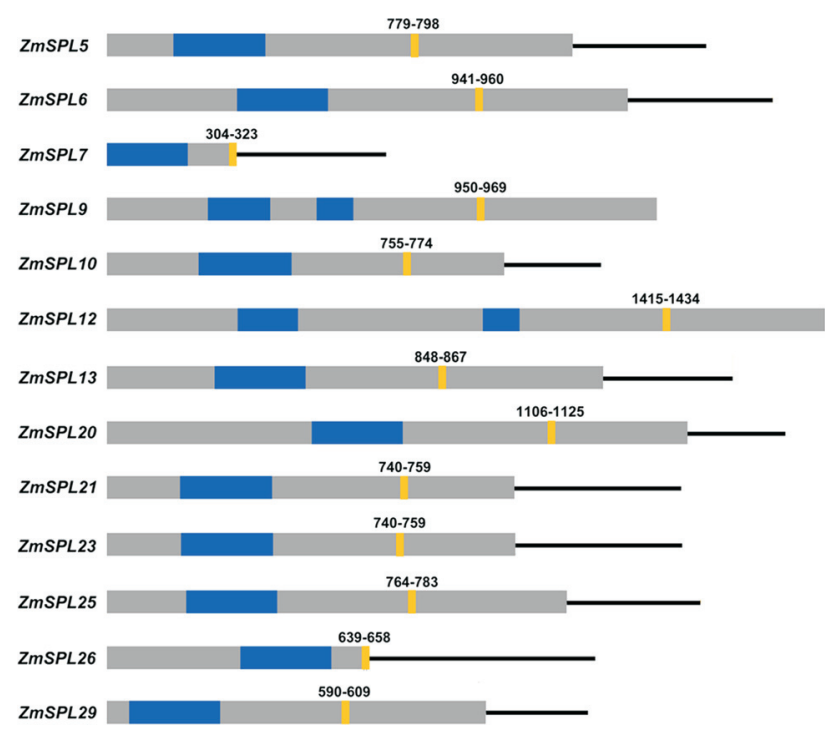

Figure 5 - ZmSPLs targeted by miR156. Open reading frames (ORFs) are indicated by grey rectangles, the SBP domain is shown by blue rectangles, and the lines flanking ORFs represent 3' UTRs. miR156 targeting sites are indicated by yellow rectangles.
$\operatorname{miR} 156$

3' CACGAGUGAGAGAAGACAGU 5'

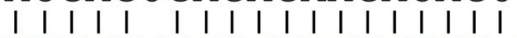

ZmSPL29 5' GUGCUCUCUCUCUUCUGUCA 3'

ZmSPL10 5' GUGCUCUCUCUCUUCUGUCA 3'

ZmSPL9 5' GUGCUCUCUCUCUUCUGUCA 3'

ZmSPL25 5' GUGCUCUCUCUCUUCUGUCA 3'

ZmSPL5 5' GUGCUCUCUCUCUUCUGUCA 3'

ZmSPL13 5' GUGCUCUCUCUCUUCUGUCA $3^{\prime}$

ZmSPL6 5' GUGCUCUCUCUCUUCUGUCA 3'

ZmSPL20 5' GUGCUCUCUCUCUUCUGUCA $3^{\prime}$

ZmSPL12 5' GUGCUCUCUCUCUUCUGUCA 3'

ZmSPL7 5' AUGCUCUCUCUCUUCUGUCA 3'

ZmSPL26 5' AUGCUCUCUCUCUUCUGUCA 3'

ZmSPL21 5'AUGCUCUCUCUCUUCUGUCA 3'

ZmSPL23 5' AUGCUCUCUCUCUUCUGUCA 3'

Figure 6 - Sequence alignments of maize miR156 with their complementary sequence in the coding sequences and 3' UTRs of ZmSPLs.

observed. For example, ZmSPL31 is only expressed in V3-Stem and SAM, while its paralog ZmSPL4 is expressed in V3-Stem and SAM, different stages of leaf and 10-DAP whole seed.

The expression patterns of the $13 \mathrm{ZmSPLs}$ targeted by miR156 were further investigated by quantitative real-time PCR (RT-qPCR) in different tissues. Four representative tissues, including root, leaf, stem, and silk were used in this study. A total of 12 genes were detected in the four tissues (ZmSPL12 was not detected), and different expression levels were found. Most of the genes showed high expression in stem or leaves, especially ZmSPL5, ZmSPL7, ZmSPL9, ZmSPL10, and ZmSPL13. We also noted that segment duplicated genes had similar expression patterns of, for example ZmSPL5 and ZmSPL13, suggesting conserved evolution in maize (Figure 8).

\section{Expression patterns of ZmSPL genes under drought stress}

While most studies so far focused on divergent biological processes regulated by SPL genes, increasing evidence indicates that SPLs have also important roles in the response to abiotic stresses (Hou et al., 2013; Wang et al., 2009). To identify the possible members of ZmSPLs involved in drought stress, the expressions of the $13 \mathrm{miR} 156$ targeted genes were further examined by RT-qPCR in maize leaves under slight, moderate, and severe stress (Figure 9). Consistent with the results of the expression at different developmental stages, the expression of ZmSPL12 was not detected, and all of the other 12 genes were responsive to drought stress, suggesting important functions in stress regulation. Among the 12 genes, the highest expression level was observed under severe stress treatment, espe- 


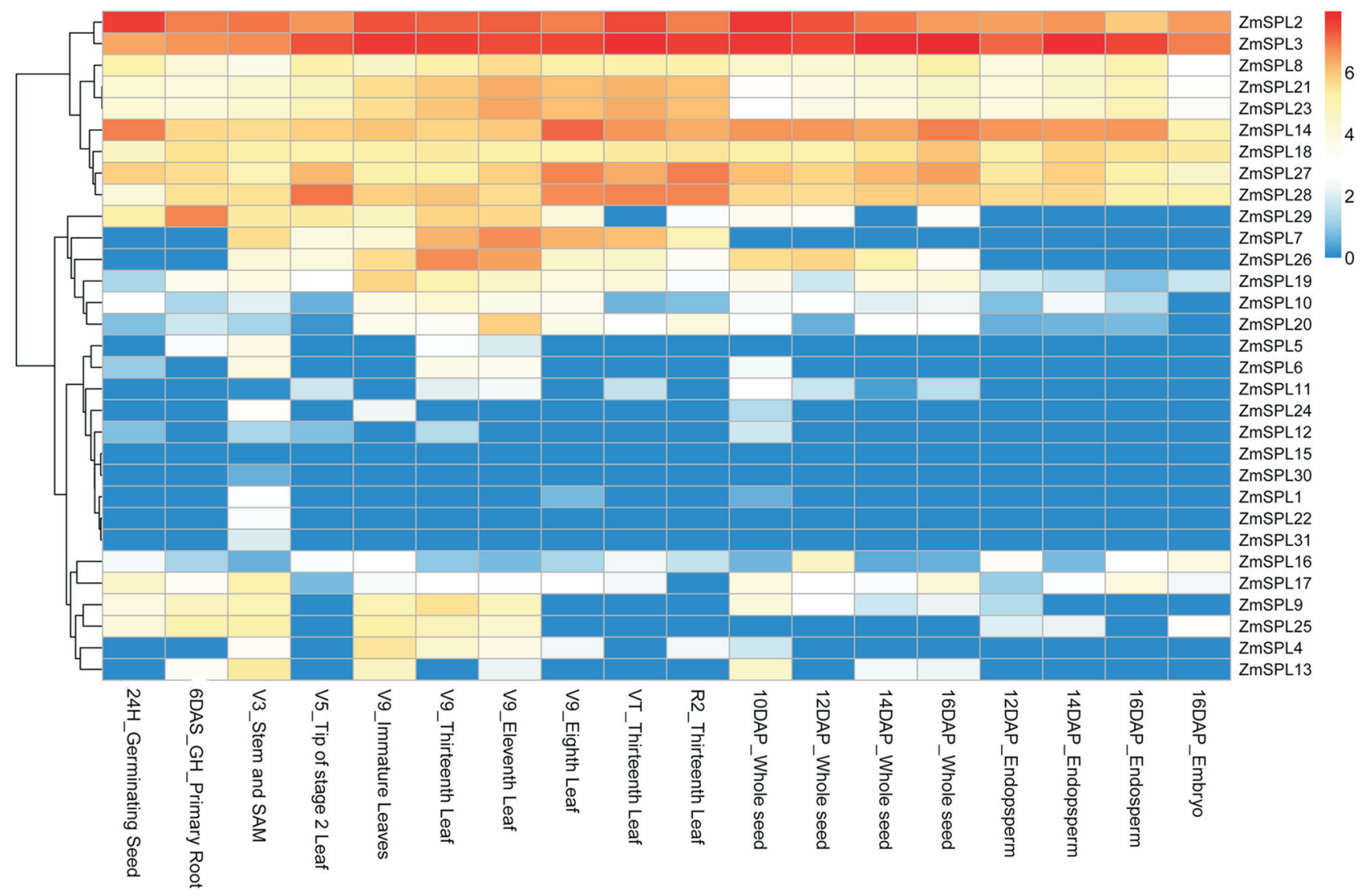

Figure 7 - Expression profiles of ZmSPLs at different developmental stages. Blue and red indicate low and high levels of transcript abundance, respectively. Tissues from different developmental stages are shown at the bottom of the heat map.

cially for ZmSPL10, -13, -21, and -26. In addition, the segment with duplicated genes showed similar expression patterns, which might suggest their redundant function in the regulation of maize drought response.

\section{Discussion}

SPLs encode a large gene family of plant-specific transcription factors that play crucial roles in plant growth and development (Klein et al., 1996; Cardon et al., 1997). In the present study, we performed a comparative analysis of the SPL family to examine the evolutionary history in different species, thus providing a foundation for gene function analysis. At least 16 SPLS were reported in Arabidopsis, 19 in rice, and 28 in Populus (Cardon et al., 1999; Xie et al., 2006; Li and Lu, 2014). In this study, a total of 31 and 18 SPLs were identified in maize and sorghum, respectively. The phylogenetic tree of the $84 \mathrm{SPL}$ proteins, including 31 of maize, 19 of rice, 18 of sorghum, and 16 of Arabidopsis, were divided into six groups. It should be noted that the number of maize SPLs was much higher than that in the mentioned species. With the purpose of elucidating the expansion mechanism of the maize SPL family, gene duplication events were investigated, which are thought to have occurred during the process of evolution. Generally, gene duplications were major driving for- ces in the evolution of genomes, and played vital roles in the expansion of gene families in various species (Moore and Purugganan, 2003; Mehan, 2004; Cannon et al., 2004), such as NBS, HD-Zip, PHD, and others (Zhao et al., 2011; Cheng et al., 2012; Wang et al., 2015).

According to the phylogenetic relationships and synteny analysis, a total of 18 segmental duplicate gene pairs of maize SPLs were identified, which accounted for $77.4 \%$ of maize SPL family genes. However, only $50 \%$ and $57.8 \%$ of the sorghum and rice $S P L s$, respectively, were detected to be involved in segmental duplication. Among the 68 SPLS of the three species, no tandem duplication events were detected. Thus, the segmental duplication was largely responsible for the expansion of SPL gene family. By comparing the frequency of segmental duplication in the three species, the segmental duplication of maize SPLs was seen to be more prevalent than in the sorghum and rice genomes, which provided a possible reason or explanation for why the numbers of SPLS are significantly different among maize, rice, and sorghum. In general, tandem duplication often occurred in rapidly evolving gene families, while segmental duplication was commonly reported in more slowly evolving gene families, e.g. the HD-Zip gene family (Cannon et al., 2004; Guo et al., 2008; Zhao et al., 2011). We concluded that the prevalence of segmental duplication 

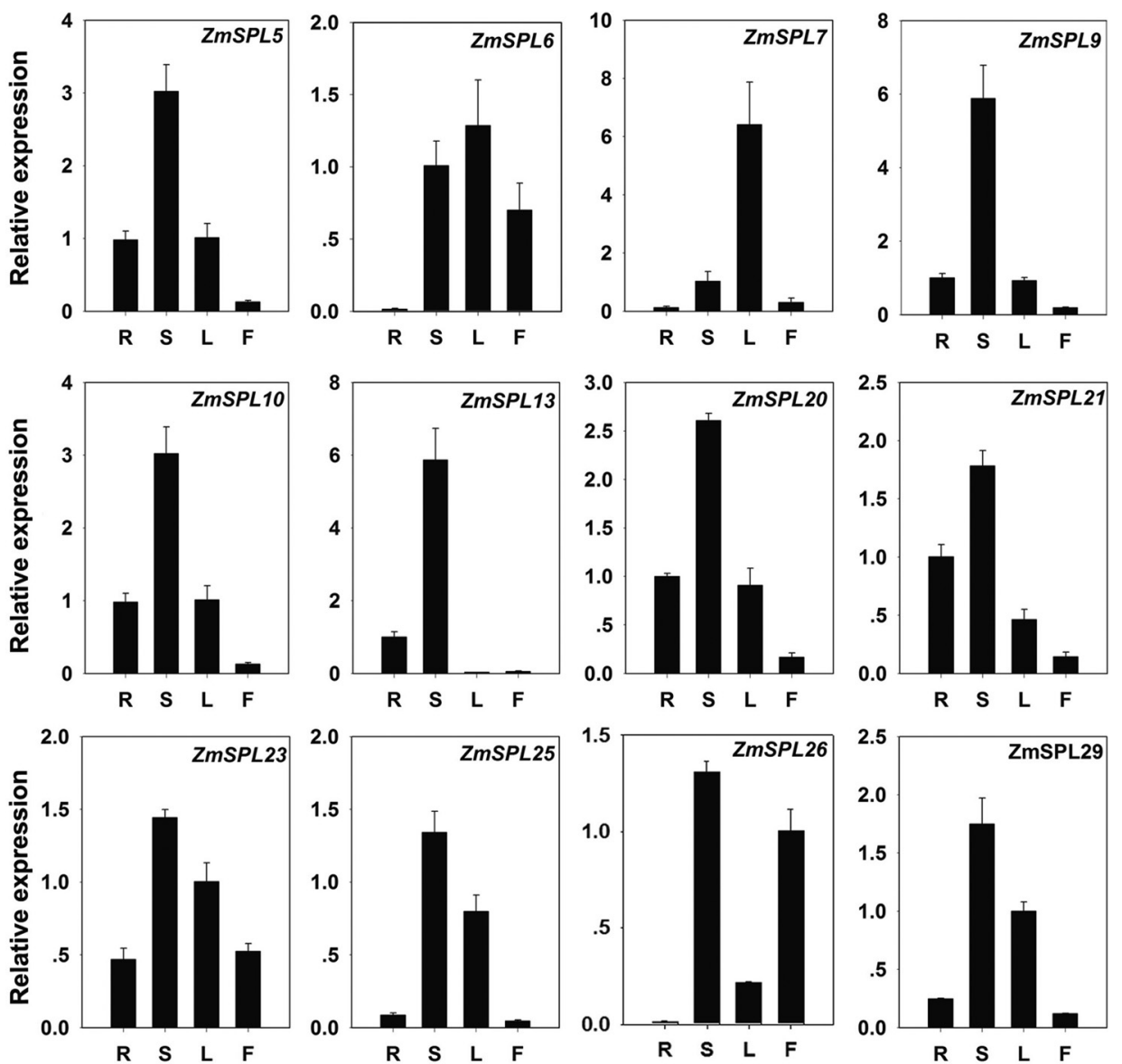

Figure 8 - Expression patterns of 12 miR156 targeted ZmSPLs in four representative tissues. R: root; S: stem; L: leaf; F: filament. Shown are means \pm SE.

demonstrated the slow evolutionary rate of the SPL gene family. In fact, a total of 38 orthologous gene pairs were identified between maize and rice, which was similar with the result between maize and sorghum (36), as well as between rice and sorghum (30). Therefore, these results suggested that the SPL gene family is a highly conserved and slowly evolving family in plants.

Whole-genome duplication (WGD) played crucial roles in plant diversification and evolution, and was often accompanied by polyploidization and gene loss (Otto and Whitton, 2000; Soltis et al., 2009). Previous studies showed that grass species have undergone several rounds of WGD. For example, maize experienced an ancient duplication prior to the divergence of grasses at approximately 50-70 Mya and a additional WGD at approximately 5 Mya, which separated maize from sorghum (Gaut, 2002; Salse et al., 2008; Schnable et al., 2009). The duplication time for the $18 \mathrm{ZmSPL}$ segmental duplication pairs ranged from 4.81 to 50.08 Mya. Among them, seven pairs showed a duplication time of less than 10 Mya. However, all the segmental duplication events in the rice and sorghum genomes were shown to have occurred between 34.46 to 47.00 Mya. These results suggested that some segmental gene pairs of maize SPLS are due to a recent duplication. In addition, se- lection pressure analysis indicated that $50 \%$ of the maize duplicated pairs evolved under positive selection. Unlike in maize, SPL gene pairs of rice and sorghum mainly evolved under purifying selection, indicating novel evolutionary features of maize SPLS.

miR156 is one of the miRNA families that is highly conserved and functions in diverse processes associated with growth and development. It has been shown to mediate posttranscriptional regulation for a subset of SPLs through direct cleavage (Wu et al., 2009; Yu et al., 2010). For example, previous studies have identified 10, 11, and 18 potential SPLs as the targets of miR156 in rice, Populus, and tomato, respectively (Wu and Poethig, 2006; Xie et al., 2006; Addoquaye et al., 2008; Schwarz et al., 2008; Li and $\mathrm{Lu}, 2014)$. In this study, 13 of $31 \mathrm{ZmSPLs}$ contained miR156 recognition sites. It is noteworthy that ZmSPL1 and $Z m S P L 17$ are not regulated by miR156, while their duplicated genes ZmSPL13 and ZmSPL20 are targets of miR156. This finding suggested that some distinct regulatory mechanisms might exist in these duplicated genes. In most cases, the miR156-regulated SPLs are master regulators that play divergent and redundant roles in plant morphology and development (Schwab, 2012). For example, AtSPL3, AtSPL4, and AtSPL5 are mainly involved in the 

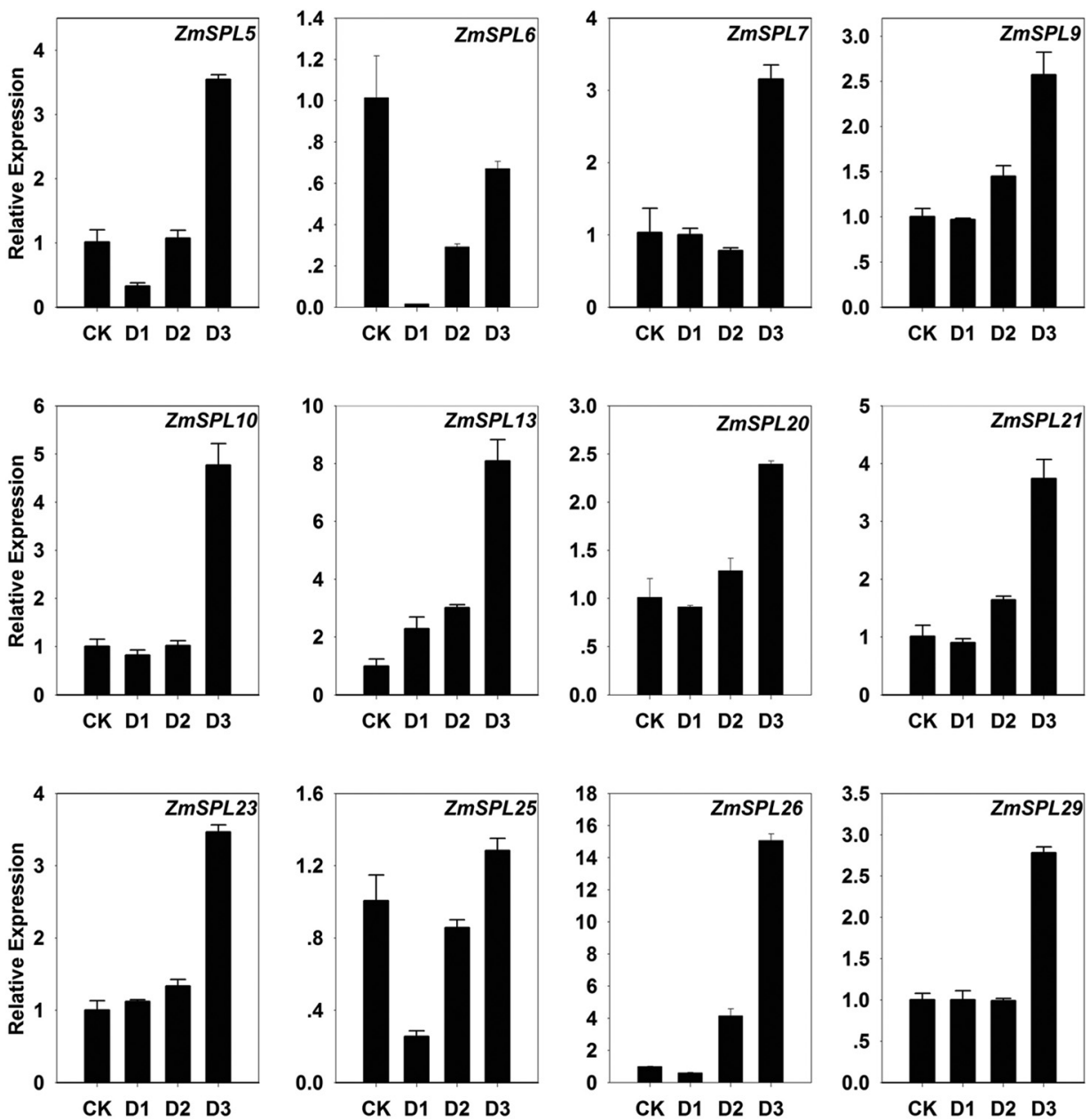

Figure 9 - Expression patterns of 12 miR156 targeted ZmSPLs under drought stress. CK: normal plant; D1: slight stress; D2: moderate stress; D3: severe stress. Shown are means \pm SE.

regulation of floral development (Cardon et al., 1997; Jung et al., 2011), while AtSPL2, AtSPL10, and AtSPL11 have been shown to be involved in lateral organ development in the reproductive phase (Shikata et al., 2009). However, whether the miR156-regulated ZmSPLs have similar regulatory roles remains to be further confirmed experimentally.

According to the microarray expression profile analysis, we found that some duplicated gene pairs have similar expression patterns, suggesting that the duplicated genes might have redundant functions in plant growth and development. Exceptions to this were also observed. The phylogenetic analysis showed that most of the maize SPL duplicated gene pairs located in the same branch had a high bootstrap value, and the duplicated gene pairs also exhibited similar exon/intron distribution and motif components. However, some duplicated gene pairs were shown to have significant divergence in expression patterns, such as ZmSPL31 and ZmSPL4. These results suggested that most of the duplicated gene pairs were still conserved in their evolution, but that functional diversification has also ac- companied the evolutionary process, as a major feature of retained duplicated genes in long-term evolution (Blanc and Wolfe, 2004). The expression patterns of the 12 miR156-targeted genes were further investigated at different developmental stages by RT-qPCR. Among the 12 $Z m S P L s$, high expression was detected in leaf and stem. Especially, the results confirmed that some segment duplicated genes have similar expression patterns, suggesting their conserved evolution and redundant functions. The expression of the $12 \mathrm{ZmSPLs}$ under drought stress was also examined. Since most of the studies about SPL family were related to developmental and biological processes, this result provided important information that the $12 \mathrm{miR} 156$ targeted genes are involved in drought stress, which may have important implications in revealing the function and mechanism of $S P L$ in the stress response.

With the advances of sequencing technologies, many new miRNAs have been identified, and an increasing number of studies on miRNAs are being reported. miR156based regulation of SPL genes participates in various biological pathways and has been reported in many plants, 
such as Arabidopsis, rice and others, but nearly no research is reported in maize. Based on our experimental results, we have identified several drought-response genes and cloned them, and this will be further studied by transgenic technology. In addition, we are verifying the actual regulatory relationship between miRNA156 and these cloned genes by 5' RACE technology and degradation group sequencing technology, and we hope our research will reveal a new molecular mechanism in the maize abiotic stress response.

\section{Acknowledgments}

This research was supported by the Natural Science Foundation of Anhui Province (NO. 1908085QC133 and 1908085QC134), the Science and Technology Major Project of Anhui Province (NO. 18030701180), and the National Natural Science Foundation of China (NO. 31540042 and 91435110). We thank the members of bioinformatics group of the Key Laboratory of Crop Biology of AnHui province for their assistance in this study.

\section{Conflict of interest}

The authors declare that they have no conflict of interest.

\section{Author contributions}

XJP, QQW and QM conceived and designed the study; XJP, QQW, YZ, XYL and QM conducted the experiments; XJP, QQW, YZ and XYL analyzed the data; XJP, QQW and QM wrote the manuscript; all authors read and approved the final version.

\section{References}

Addoquaye C, Eshoo TW, Bartel DP and Axtell MJ (2008) Endogenous siRNA and miRNA targets identified by sequencing of the Arabidopsis degradome. Curr Biol 18:758.

Axtell MJ and Bowman JL (2008) Evolution of plant microRNAs and their targets. Trends Plant Sci 13:343-349.

Bailey TL and Elkan C (1995) The value of prior knowledge in discovering motifs with MEME. Proc Int Conf Intell Syst Biol 3:21-29.

Birkenbihl RP, Jach G, Saedler H and Huijser P (2005) Functional dissection of the plant-specific SBP-domain: Overlap of the DNA-binding and nuclear localization domains. J Mol Biol 352:585-596.

Blanc G and Wolfe KH (2004) Widespread paleopolyploidy in model plant species inferred from age distributions of duplicate genes. Plant Cell 16:1667-1678.

Cannon SB, Mitra A, Baumgarten A, Young ND and May G (2004) The roles of segmental and tandem gene duplication in the evolution of large gene families in Arabidopsis thaliana. BMC Plant Biol 4:10.

Cardon GH, Höhmann S, Nettesheim K, Saedler H and Huijser P (1997) Functional analysis of the Arabidopsis thaliana SBP-box gene SPL3 : A novel gene involved in the floral transition. Plant J 12:367-377.
Cardon G, Höhmann S, Klein J, Nettesheim K, Saedler H and Huijser P (1999) Molecular characterisation of the Arabidopsis SBP-box genes. Gene 237:91-104.

Cheng Y, Li X, Jiang H, Wei M, Miao W, Yamada T and Ming Z (2012) Systematic analysis and comparison of nucleotidebinding site disease resistance genes in maize. FEBS J 279:2431-2443.

Chuck G, Whipple C, Jackson D and Hake S (2010) The maize SBP-box transcription factor encoded by tasselsheath 4 regulates bract development and the establishment of meristem boundaries. Development 137:1243-1250.

Dai X and Zhao PX (2011) psRNATarget: A plant small RNA target analysis server. Nucleic Acids Res 39:W155.

Dorweiler JE (2008) Feminized tassels of maize mop 1 and $t s 1 \mathrm{mu}-$ tants exhibit altered levels of miR156 and specific SBP-box genes. Planta 229:99-113.

Edgar RC (2004) MUSCLE: Multiple sequence alignment with high accuracy and high throughput. Nucleic Acids Res 32:1792-1797.

Finn RD, Mistry J, Schusterböckler B, Griffiths-Jones S, Hollich V, Lassmann T, Moxon S, Marshall M, Khanna A and Durbin R (2006) Pfam: Clans, web tools and services. Nucleic Acids Res 34: 247-251.

Gaut BS (2002) Evolutionary dynamics of grass genomes. New Phytol 154:15-28.

Gaut BS, Morton BR, Mccaig BC and Clegg MT (1996) Substitution rate comparisons between grasses and palms: synonymous rate differences at the nuclear gene Adh parallel rate differences at the plastid gene $r b c L$. Proc Natl Acad Sci USA 93:10274-110279.

Guo AY, Zhu QH, Gu X, Ge S, Yang J and Luo J (2008) Genome-wide identification and evolutionary analysis of the plant specific SBP-box transcription factor family. Gene 418:1-8.

Hou H, Li J, Gao M, Singer SD, Wang H, Mao L, Fei Z and Wang X (2013) Genomic organization, phylogenetic comparison and differential expression of the SBP-box family genes in grape. PLoS One 8:e59358.

Hu B, Jin J, Guo AY, Zhang H, Luo J and Gao G (2015) GSDS 2.0: An upgraded gene feature visualization server. Bioinformatics 31:1296.

Jones-Rhoades MW, Bartel DP and Bartel B (2006) MicroRNAs and their regulatory roles in plants. Annu Rev Plant Biol 57:19-53.

Jung JH, Seo PJ, Kang SK and Park CM (2011) miR172 signals are incorporated into the miR156 signaling pathway at the SPL3/4/5 genes in Arabidopsis developmental transitions. Plant Mol Biol 76:35-45.

Klein J, Saedler H and Huijser P (1996) A new family of DNA binding proteins includes putative transcriptional regulators of the Antirrhinum majus floral meristem identity gene SQUAMOSA. Mol Genet Genomics 250:7-16.

Kozomara A and Griffiths-Jones S (2010) miRBase: Integrating microRNA annotation and deep-sequencing data. Nucleic Acids Res 39:D152-D157.

Krzywinski M, Schein J, Birol I, Connors J, Gascoyne R, Horsman D, Jones SJ and Marra MA (2009) Circos: An information aesthetic for comparative genomics. Genome Res 19:1639-1645. 
Lee RC, Feinbaum RL and Ambros V (1993) The C. elegans heterochronic gene lin-4 encodes small RNAs with antisense complementarity to lin-14. Cell 75:843-854.

Letunic I (2009) SMART 6: Recent updates and new developments. Nucleic Acids Res 37:D229.

Li C and Lu S (2014) Molecular characterization of the SPL gene family in Populus trichocarpa. BMC Plant Biol 14:131.

Lynch M and Conery JS (2000) The evolutionary fate and consequences of duplicate genes. Science 290:1151-1155.

Manning K, Tör M, Poole M, Hong Y, Thompson AJ, King GJ, Giovannoni JJ and Seymour GB (2006) A naturally occurring epigenetic mutation in a gene encoding an SBP-box transcription factor inhibits tomato fruit ripening. Nature Genetics 38:948-952.

Mehan MR (2004) A genome-wide survey of segmental duplications that mediate common human genetic variation of chromosomal architecture. Hum Genomics 1:335-344.

Moore RC and Purugganan MD (2003) The early stages of duplicate gene evolution. Proc Natl Acad Sci USA 100:1568215687.

Moreno MA, Harper LC, Krueger RW, Dellaporta SL and Freeling M (1997) liguleless1 encodes a nuclear-localized protein required for induction of ligules and auricles during maize leaf organogenesis. Genes Dev 11:616-628.

Otto SP and Whitton J (2000) Polyploid incidence and evolution. Annu Rev Genet 34:401-437.

Peng X, Zhao Y, Cao J, Zhang W, Jiang H, Li X, Ma Q, Zhu S and Cheng B (2012) CCCH-type zinc finger family in maize: Genome-wide identification, classification and expression profiling under abscisic acid and drought treatments. PLoS One 7:e40120.

Quraishi UM, Abrouk M, Murat F, Pont C, Foucrier S, Desmaizieres $\mathrm{G}$, Confolent $\mathrm{C}$, Rivière $\mathrm{N}$, Charmet $\mathrm{G}$ and Paux $\mathrm{E}$ (2011) Cross-genome map based dissection of a nitrogen use efficiency ortho-metaQTL in bread wheat unravels concerted cereal genome evolution. Plant J 65:745-756.

Rhoades MW, Reinhart BJ, Lim LP, Burge CB, Bartel B and Bartel DP (2002) Prediction of plant microRNA targets. Cell 110:513-520.

Riechmann JL, Heard J, Martin G, Reuber L, Jiang C, Keddie J, Adam L, Pineda O, Ratcliffe OJ and Samaha RR (2001) Arabidopsis transcription factors: Genome-wide comparative analysis among eukaryotes. Science 290:2105-2110

Ross I and Robert G (2008) R: A language and environment for statistical computing. R Foundation for Statistical Computing. Computing 1:12-21.

Rozas J, Sánchezdelbarrio JC, Messeguer X and Rozas R (2003) DnaSP, DNA polymorphism analyses by the coalescent and other methods. Bioinformatics 19:2496-2497.

Salse JM, Bolot S, Throude ML, Jouffe V, Piegu BT, Quraishi UM, Calcagno T, Cooke R, Delseny M and Feuilleta C (2008) Identification and characterization of shared duplications between rice and wheat provide new insight into grass genome evolution. Plant Cell 20:11-24.

Schnable PS, Ware D, Fulton RS, Stein JC, Wei F, Pasternak S, Liang C, Zhang J, Fulton L and Graves TA (2009) The B73 maize genome: Complexity, diversity, and dynamics. Science 326:1112-1115.

Schwab R (2012) The roles of miR156 and miR172 in phase change regulation. In: Sunkar R (ed) MicroRNAs in Plant
Development and Stress Responses. Springer, Berlin, pp 49-75.

Schwab R, Palatnik JF, Riester M, Schommer C, Schmid M and Weigel D (2005) Specific effects of microRNAs on the plant transcriptome. Dev Cell 8:517-527.

Schwarz S, Grande AV, Bujdoso N, Saedler H and Huijser P (2008) The microRNA regulated SBP-box genes SPL9 and SPL15 control shoot maturation in Arabidopsis. Plant Mol Biol 67:183-195.

Sekhon RS, Briskine R, Hirsch CN, Myers CL, Springer NM, Buell CR, Leon ND and Kaeppler SM (2013) Maize gene atlas developed by RNA sequencing and comparative evaluation of transcriptomes based on RNA sequencing and microarrays. PLoS One 8:e61005.

Shikata M, Koyama T, Mitsuda N and Ohme-Takagi M (2009) Arabidopsis SBP-box genes SPL10, SPL11 and SPL2 control morphological change in association with shoot maturation in the reproductive phase. Plant Cell Physiol 50:2133-2145.

Soltis DE, Albert VA, Leebens-Mack J, Bell CD, Paterson AH, Zheng C, Sankoff D, Depamphilis CW, Wall PK and Soltis PS (2009) Polyploidy and angiosperm diversification. Am J Bot 96:336-348.

Stone JM, Liang X, Nekl ER and Stiers JJ (2005) Arabidopsis AtSPL14, a plant-specific SBP-domain transcription factor, participates in plant development and sensitivity to fumonisin B1. Plant J 41:744-754.

Tamura K (2007) MEGA4: Molecular Evolutionary Genetics Analysis (MEGA) software version 4.0. Mol Biol Evol. 24:1596-1599.

Unte US, Sorensen AM, Pesaresi P, Gandikota M, Leister D, Saedler H and Huijser P (2003) SPL8, an SBP-box gene that affects pollen sac development in Arabidopsis. Plant Cell 15:1009-1019.

Wang H, Nussbaumwagler T, Li B, Zhao Q, Vigouroux Y, Faller M, Bomblies K, Lukens L and Doebley JF (2005) The origin of the naked grains of maize. Nature 436:714-719.

Wang JW, Czech B and Weigel D (2009) miR156-regulated SPL transcription factors define an endogenous flowering pathway in Arabidopsis thaliana. Cell 138:738-749.

Wang Q, Liu J, Wang Y, Zhao Y, Jiang H and Cheng B (2015) Systematic analysis of the maize PHD-finger gene family reveals a subfamily involved in abiotic stress eesponse. Int $\mathrm{J}$ Mol Sci 566:95-108

Wang Y, Tang H, Debarry JD, Tan X, Li J, Wang X, Lee TH, Jin H, Marler B and Guo H (2012) MCScanX: a toolkit for detection and evolutionary analysis of gene synteny and collinearity. Nucleic Acids Research 40:e49

Wu G, Park MY, Conway SR, Wang JW, Weigel D and Poethig RS (2009) The sequential action of miR156 and miR172 regulates developmental timing in Arabidopsis. Cell 138:750-759.

Wu G and Poethig RS (2006) Temporal regulation of shoot development in Arabidopsis thaliana by miR156 and its target SPL3. Development 133:3539-3547.

Xie K, Wu C and Xiong L (2006) Genomic organization, differential expression, and interaction of SQUAMOSA promoterbinding-like transcription factors and microRNA156 in rice. Plant Physiol 142:280-293. 
Xing SP (2010) miR156-targeted and nontargeted SBP-Box transcription factors act in concert to secure male fertility in Arabidopsis. Plant Cell 22: 3935-3950.

Yamasaki K, Kigawa T, Inoue M, Tateno M, Yamasaki T, Yabuki T, Aoki M, Seki E, Matsuda T and Nunokawa E (2004) A novel zinc-binding motif revealed by solution structures of DNA-binding domains of Arabidopsis SBP-family transcription factors. J Mol Biol 337:49-63.

Yu N, Cai WJ, Wang S, Shan CM, Wang LJ and Chen XY (2010) Temporal control of trichome distribution by microRNA156-targeted SPL genes in Arabidopsis thaliana. Plant Cell 22:2322-2335.

Zhang B, Pan X, Cobb GP and Anderson TA (2006) Plant microRNA: A small regulatory molecule with big impact. Dev Biol 289:3-16.

Zhang Y, Schwarz S, Saedler H and Huijser P (2007) SPL8, a local regulator in a subset of gibberellin-mediated developmental processes in Arabidopsis. Plant Mol Biol 63:429439.

Zhao Y, Zhou Y, Jiang H, Li X, Gan D, Peng X, Zhu S and Cheng B (2011) Systematic analysis of sequences and expression patterns of drought-responsive members of the HD-Zip gene family in maize. PLoS One 6:e28488.

\section{Supplementary material}

The following online material is available for this article:

Figure S1 - Phylogenetic relationships and gene structure of the ZmSPLs.

Figure S2 - Distribution of conserved motifs identified in the putative SPL proteins.

Figure S3 - Chromosomal locations of ZmSPLs on the 10 maize chromosomes.

Table S1 - List of gene-specific primers used in the present study.

Table S2 - Detailed information on the 31 SPLs in the maize genome.

lable S3 - Detailed information on the 18 sorghum SPLS. Table S4 - Information about orthologous genes in maize, rice, and sorghum.

Table S5 - Detailed information on the 20 motifs identified in $Z m S P L s$.

Associate Editor: Adriana S. Hermely

License information: This is an open-access article distributed under the terms of the Creative Commons Attribution License (type CC-BY), which permits unrestricted use, distribution and reproduction in any medium, provided the original article is properly cited. 